\title{
Dysfunction and Restoration of Endothelial Cell Communications in Pulmonary Arterial Hypertension: Therapeutic Implications
}

\author{
Christophe Guignabert
}

\begin{abstract}
Impairment of the pulmonary endothelial communications contributes to the development and progression of pulmonary arterial hypertension (PAH). Investigations currently under way are likely to produce novel therapeutic strategies that target pulmonary endothelial dysfunction linked to PAH. This chapter outlines the complex role of the dysfunctional pulmonary endothelium in PAH and its potential value as a target for innovative therapies.
\end{abstract}

\section{Keywords}

Pulmonary hypertension · Pathogenesis · Endothelial dysfunction · Cell-cell communication - Therapeutic target

\subsection{Introduction}

Pulmonary arterial hypertension (PAH) is a severe and progressive cardiopulmonary disorder characterized by non-specific symptoms (i.e., breathlessness, fatigue, weakness, angina, and syncope) that take years before appearing, usually after the disease is in an advanced stage. In Europe, PAH prevalence and incidence are in the range of 15-60 subjects per million population and 5-10 cases per million per year, respectively. Despite recent progress in the treatment of PAH, most patients still die from the disease or fail to respond adequately to medical therapy with a 5-year survival of $59 \%$. Current treatments can relieve some PAH symptoms and slow the progress of

\section{Guignabert ( $\square)$}

INSERM UMR_S 999, Hôpital Marie Lannelongue, Le Plessis-Robinson, France

Faculté de Médecine, Université Paris-Sud, Université Paris-Saclay,

Le Kremlin-Bicêtre, France

e-mail: christophe.guignabert@inserm.fr 
the disease in some patients, but they have limited impact on the progressive pulmonary vascular remodeling that eventually culminates in right heart failure [1].

Pulmonary endothelial dysfunction is a critical contributor that could be detrimental for the onset and progression of this severe cardiopulmonary disorder [2-4]. Therefore, early diagnosis and better understanding of the mechanisms underlying the altered pulmonary endothelial cell (EC) phenotype and endothelial communications with both resident vascular cells (smooth muscle cells, fibroblasts, myofibroblasts, and pericytes) and inflammatory cells are needed to propose and develop new, more adapted and more powerful therapeutic tools.

\subsection{Pulmonary Endothelial Dysfunction and the Pathobiology of PAH}

At the interface between bloodstream and lung tissue, the pulmonary ECs play critical roles not only in optimizing gas exchanges but also in the local adaptations of vascular tone, in the maintenance of a thrombosis-free surface, in the control of inflammatory cell adhesion and trafficking, as well as in the maintenance of the vascular wall integrity and for normal angiogenesis. Importantly, they are dynamic and adapt their roles and phenotypes according to the nature of the local milieu (Fig. 19.1). Indeed, the pulmonary ECs are highly metabolically active, sensing and responding to signals from extracellular environments by secreting the correct substance(s) by which it may maintain the vasomotor balance and vascular-tissue homeostasis [4].

As a vital part of the pulmonary vascular wall, alterations of the pulmonary endothelium are now well established to be central role in the pathogenesis of PAH. The main characteristics of pulmonary endothelial alterations or dysfunction in PAH includes, among others, a reduction in the secretion of vasodilator molecules such as prostacyclin $\left(\mathrm{PGI}_{2}\right)$ and nitric oxide (NO), and an increase in the potent vasoconstrictor endothelin (ET)-1 [5]. In addition, our group has highlighted that pulmonary ECs from PAH patients exhibit a pro-inflammatory phenotype characterized by an increased

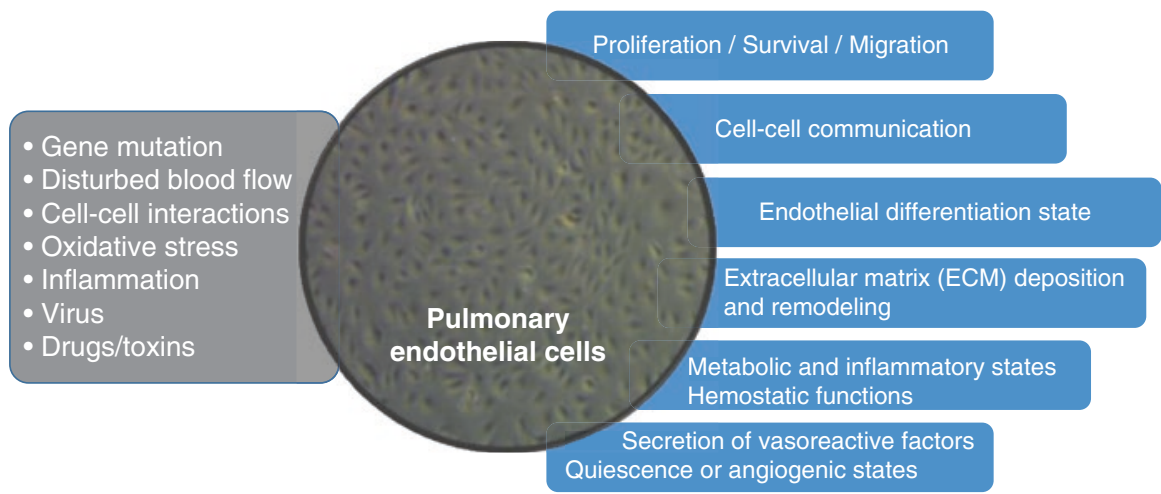

Fig. 19.1 The pulmonary vascular endothelium is a multifunctional organ and is critically involved in modulating vascular tone and structure. Through their capacity to spatially segregate and temporally integrate a diverse range of extracellular signals (in gray), pulmonary ECs adapt their roles and phenotype (in blue) according to the nature of the local milieu 
expression of key adhesion molecules (E-selectin, ICAM-1, and VCAM-1) together with an excessive secretion of different inflammatory mediators (such as interleukin (IL)-1 $\alpha$, IL-6, IL-8, IL-12, macrophage migration inhibitory factor (MIF), and CCL2) [6]. Pulmonary ECs from PAH patients also exhibit metabolic alterations including heightened glycolysis [7]. Finally, decreased capacities to form vascular tube in vitro [8] and acquisition of some mesenchymal markers have also been reported [9]. Finally, a pro-proliferative and apoptosis-resistant phenotype has been described [10-12]. Various stimuli such as high glucose, insulin resistance, disturbed blood flow, and oxidative stress can lead to endothelial dysfunction. Therefore, further research is needed to elucidate their specific contribution to the altered pulmonary EC phenotype in PAH.

Importantly, this excessive local secretion of key factors by the dysfunctional pulmonary endothelium in PAH has also been demonstrated to play a critical role in modulating the behavior and functions of adjacent vascular cells (smooth muscle cells, fibroblasts myofibroblasts, and pericytes) and several immune cells that infiltrate the remodeled pulmonary vessels, thereby contributing to the vascular remodeling in PAH [3] (Fig. 19.2). Although considerable progress has been made, the
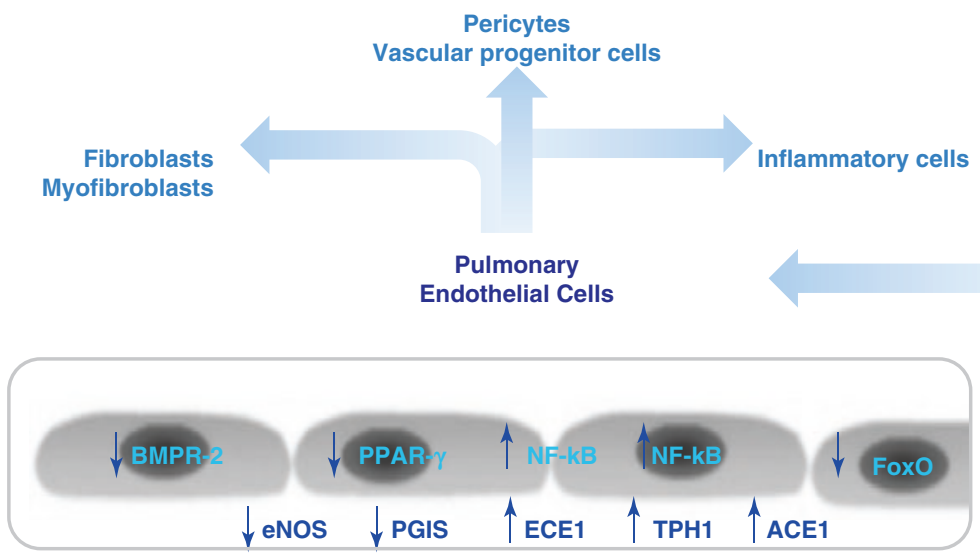

$\downarrow$ NO $\downarrow$ Apelin $\downarrow$ Prostacyclin $\uparrow$ ET-1 $\uparrow$ 5-HT $\uparrow$ FGF2 $\uparrow$ Angll $\uparrow_{\text {LL-6 }} \uparrow$ Leptin $\uparrow$ SDF-1 $\uparrow$ MIF APJ Receptor ET-A 5-HTT/5HT2A/5HT2B FGFR2 AT1R IL6R/gp130 ObR CXCR

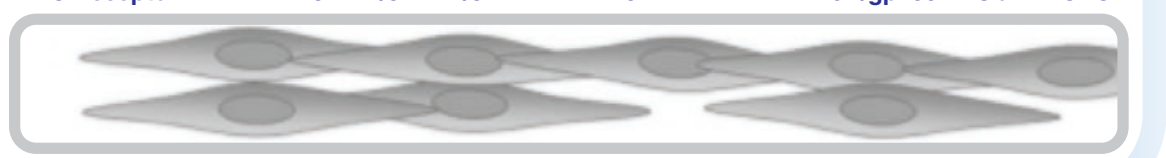

Vascular Smooth Muscle Cells

Fig. 19.2 Importance of endothelial miscommunication in PAH. In PAH, the dynamic and unadapted pulmonary vascular remodeling is partly driven by several genetic and environmental factors and also by the presence of different intrinsic abnormalities within the pulmonary ECs that include, among others, a loss and/or dysfunctions of the BMPR-2 signaling, decreased PPAR- $\gamma$ and FoxO signaling, and constitutive activations of NF- $\mathrm{KB}$ and/or PHD2/HIF axes. Consequently, the altered pulmonary ECs create a permissive pericellular/extracellular environment for the progression of the pulmonary vascular remodeling, mainly because they miscommunicate with both resident vascular cells (smooth muscle cells, fibroblasts, myofibroblasts, and pericytes) and inflammatory cells mainly through the secretion of different key molecules 
triggers, the mechanisms, and the consequences of dysfunctional pulmonary endothelium in PAH are still not completely understood. A better knowledge of these key aspects would help finding new PAH biomarkers and/or novel therapeutic targets to reverse or even stop the progression of this irreversible vascular remodeling.

\subsection{Current Promising Strategies for Restoring Pulmonary Endothelial Dysfunction and Cell-Cell Communications}

Below, a few of the most promising therapeutic strategies that target the dysfunctional pulmonary endothelium are underlined.

\subsubsection{Restoring the Balance of Vasodilation and Vasoconstriction}

In PAH, the discovery of abnormalities affecting the endothelial production and secretion of the three main mediators (NO, PGI2 and ET-1) or defects in their signaling pathways involved in the regulation of pulmonary vasoreactivity has led to the development of currently approved PAH therapies which have drastically improved the quality of life and clinical outcomes in PAH patients. Major therapeutic advances are also leading to the development of new molecules in these three pathways characterized by better pharmaceutical properties, such as longer half-life, increased receptor affinity, prolonged receptor binding, and routes of administration easier to manage. Nevertheless, other vasoreactive mediators are known to be involved and can represent new therapeutic targets, such as RhoA/ROCK inhibitors, anti-serotoninergic drugs, apelin agonists, and inhibitors or blockers of the ACE (angiotensin-converting enzyme)-1/angiotensin (Ang)-II/AT1 receptor, agents that stimulate the ACE2-Ang-(1-7)-Mas pathway [13].

Beyond vasoconstrictors and vasodilators, the recent identification of familial cases of PAH related to heterozygous missense variants in the $K C N K 3$ gene (encoding TWIK-related acid-sensitive potassium channel (TASK)-1) has revived interest in the concept of channelopathy in PAH [14]. The main challenge is this area is to identify specific small molecules to restore the activity of these ion channels in the dysfunctional pulmonary endothelium in PAH. Undoubtedly, this strategy will have significant impact for future investigations in the field and in other cardiovascular disorders.

\subsubsection{Restitution of the Defective BMPR-2 Signaling System}

Several notions support that enhancement of the BMPR-2 axis pulmonary vascular cells could offer a novel approach for the treatment of PAH $[15,16]$. Consistent with this observation, preliminary data suggested that low-dose of tacrolimus (FK506) is well tolerated and increases BMPR-2 in subsets of PAH patients [17]. Based on these findings, a phase II randomized control trial in PAH has been initiated in stable PAH patients (NCT01647945). Interestingly, promising findings have also been obtained with exogenous BMP9 and ataluren, a drug that induces ribosomal read through of nonsense 
mutations, and thus may offer the possibility of future therapies [18, 19]. Nevertheless, further studies are needed to validate these hypotheses [20]. Indeed, the low penetrance of the disease among BMPR 2 mutation carriers suggests that additional genetic or environmental factors are required for the development of PAH [15]. In addition, it has been recently shown that the selective BMP9 loss or inhibition does not predispose, but partially prevent or protect against experimental pulmonary hypertension. Therefore, the apparent ambivalent role of the BMP9/BMPR-2 signaling pathway should stimulate work to better understand this feature and the underlying mechanisms.

\subsubsection{Targeting Cell Proliferation and Cell accumulation}

In PAH, several transcription factors [such as peroxisome proliferator-activated receptor (PPAR- $\gamma$ ), signal transducer and activator of transcription (STAT)-3, HIPPO, FoxO1, and hypoxia-inducible factor (HIF)] and growth factors are known to be overexpressed or overactive in the dysfunctional endothelium [5, 13]. Platelet-derived growth factor (PDGF), fibroblast growth factor (FGF)-2, epidermal growth factor (EGF), vascular endothelial growth factor (VEGF), and nerve growth factor (NGF) are key players in this regard. Furthermore, other factors and signaling may serve as potential target candidates for anti-proliferative strategies in PAH, such as the RhoA/Rho-kinase signaling system, the mammalian target of rapamycin (mTOR) and insulin-like growth factor signaling systems, connective tissue growth factor (CTGF), hepatocyte growth factor (HGF), and placental growth factor (PIGF). However, further studies on their pathogenic roles are important. Although targeting growth factors remains a relevant therapeutic strategy in $\mathrm{PAH}$, a better understanding of tyrosine kinase inhibitor (TKI) properties and characteristics, particularly for lung and heart homeostases, will help designing new drugs that specifically block PAH pathways without severe side effects.

Our group has underlined that the dysfunctional pulmonary endothelium in PAH partly contributes to the excessive pericytic envelope, notably through the overabundance of the endothelial-derived FGF-2 and IL-6 [21]. We also demonstrated that this increase in pulmonary pericyte coverage in PAH could represent a local source of smooth muscle-like cells and actively contributes to the pulmonary vascular remodeling. Pericytes are central regulators of vascular development, stabilization, maturation, and remodeling, modulating EC proliferation, vascular tone and autoimmunity. Although pulmonary pericytes could likely represent a source of promising therapeutic targets in PAH, little is known about the intrinsic characteristics of lung pericytes in PAH and their exact roles in the progression of this irreversible remodeling linked to PAH. Therefore, additional studies are needed.

\subsubsection{Restitution of an Adapted Extracellular Matrix (ECM) Remodeling}

In PAH, the dynamic and unadapted remodeling of the ECM not only leads to qualitative and quantitative changes that are important to modulate vessel stiffness, but also forms a permissive milieu that profoundly influences cell motility, proliferation, 
apoptosis, and differentiation. In addition, the excessive release of growth factors and other molecules that are encrypted in the ECM together with the exposure of functionally cryptic sites (i.e., within collagens, fibronectin, laminins, elastin) and the release of ECM fragments also have critical roles in modulating cell behavior and vascular remodeling. Beneficial effects of different strategies that restitute these ECM alterations in PAH have been reported [3].

\subsubsection{Targeting Metabolic Changes}

Several evidences suggest that a shift in energy production from mitochondrial oxidative phosphorylation to glycolysis is present in pulmonary ECs and contributes to PAH development and progression. In lines with this notion, a recent clinical trial demonstrates that inhibition of pyruvate dehydrogenase kinase (PDK), an inhibitor of the mitochondrial enzyme pyruvate dehydrogenase ( $\mathrm{PDH}$, the gatekeeping enzyme of glucose oxidation) improves $\mathrm{PAH}$ in the absence of functional variants of SIRT3 and UCP2 [22]. Other metabolic pathways and related factors also represent interesting targets for $\mathrm{PAH}$ therapy, including modulators of hypoxia-inducible factor (HIF) and the phosphoinositide 3-kinase/protein kinase $\mathrm{B} /$ mammalian target of rapamycin (PI3K/Akt/mTOR) pathway, mitochondrial phosphatase and tensin homolog (PTEN)-induced kinase 1 (PINK1), and the HIPPO and p53 signaling pathways. However, a more complete understanding of the overall risk-benefit ratio of these different strategies with long-term follow-up has to be evaluated.

\subsubsection{Targeting the Vicious Cycle Between Endothelial Dysfunction and Immune Dysregulation}

The interplay between endothelial dysfunction and immune dysregulation warrants future clinical studies of newer biologic agents that can target specific inflammatory pathways [23]. Through the expression of adhesion molecules (ICAM-1, VCAM-1, and E-selectins) and the secretion of various key cytokines and chemokines, the dysfunctional pulmonary endothelium facilitates the infiltration of inflammatory cells in the perivascular area $[6,24]$. Remarkably, we demonstrated that the dysfunctional pulmonary endothelium in PAH through its overproduction of leptin inhibits Treg cell function [25]. Consistent with this observation, our group has shown that PAH patients display normal Treg cell count but with an altered function. Interestingly, it is also known that the pulmonary vasculature is sensitive to inflammation and can respond to inflammatory stimuli by abnormal proliferation and/or migration and apoptotic-resistant phenotype. In line with these notions, we recently identified ectopic upregulation of membrane-bound IL-6 receptor (IL6R) on PA-SMCs in PAH patients and in rodent models of pulmonary hypertension and demonstrated its key role for PA-SMC accumulation in vitro and in vivo [26]. 


\subsection{Future Directions and Clinical Implications}

In summary, substantial work remains to be done to discover and/or develop a new, better tolerated, and more powerful curative treatment for PAH that combines promotion of vasorelaxation and restoration of endothelial communications. However, important discoveries in the molecular mechanisms contributing to the pulmonary endothelial dysfunction in PAH have been obtained and our knowledge continues to accelerate. Thus, our improved understanding of additional pathways in this condition will presumably lead to the development of novel therapeutic strategies that could specifically target endothelial dysfunction in the near future. A comprehensive estimation and evaluation of risks and benefits, potential ways to circumvent the risk of adverse effects on the adaptative myocardial hypertrophy, and a targeted delivery of these new drugs in a safe and effective manner are still major challenges facing clinical researchers.

Acknowledgments This work was supported by grants from the INSERM, the University of Paris-Sud and the University Paris-Saclay, the Marie Lannelongue Hospital, the ANR (ANR16-CE17-0014 Tamirah), the FRM (DEQ20150331712), and in part by the DHU TORINO, the AP-HP, Service de Pneumologie, Centre de Référence de l'Hypertension Pulmonaire Sévère, the LabEx LERMIT (ANR-10-LABX-0033), the French PAH patient association (HTAP France), and the FRSR-FdS.

\section{References}

1. Humbert M, Guignabert C, Bonnet S, Dorfmüller P, Klinger JR, Nicolls MR, Olschewski AJ, Pullamsetti SS, Schermuly RT, Stenmark KR, Rabinovitch M. Pathology and pathobiology of pulmonary hypertension: state of the art and research perspectives. Eur Respir J. 2019;53(1): 1801887. https://doi.org/10.1183/13993003.01887-2018.

2. Guignabert C, Dorfmuller P. Pathology and Pathobiology of Pulmonary Hypertension. Semin Respir Crit Care Med. 2017;38(5):571-84. https://doi.org/10.1055/s-0037-1606214.

3. Guignabert C, Tu L, Girerd B, Ricard N, Huertas A, Montani D, Humbert M. New molecular targets of pulmonary vascular remodeling in pulmonary arterial hypertension: importance of endothelial communication. Chest. 2015;147(2):529-37. https://doi.org/10.1378/ chest.14-0862.

4. Huertas A, Guignabert C, Barbera JA, Bartsch P, Bhattacharya J, Bhattacharya S, Bonsignore MR, Dewachter L, Dinh-Xuan AT, Dorfmuller P, Gladwin MT, Humbert M, Kotsimbos T, Vassilakopoulos T, Sanchez O, Savale L, Testa U, Wilkins MR. Pulmonary vascular endothelium: the orchestra conductor in respiratory diseases: Highlights from basic research to therapy. Eur Respir J. 2018;51(4). https://doi.org/10.1183/13993003.00745-2017.

5. Huertas A, Tu L, Guignabert C. New targets for pulmonary arterial hypertension: going beyond the currently targeted three pathways. Curr Opin Pulm Med. 2017;23(5):377-85. https://doi. org/10.1097/MCP.0000000000000404.

6. Le Hiress M, Tu L, Ricard N, Phan C, Thuillet R, Fadel E, Dorfmuller P, Montani D, de Man F, Humbert M, Huertas A, Guignabert C. Proinflammatory signature of the dysfunctional endothelium in pulmonary hypertension. Role of the macrophage migration inhibitory factor/ CD74 complex. Am J Respir Crit Care Med. 2015;192(8):983-97. https://doi.org/10.1164/ rccm.201402-0322OC. 
7. Diebold I, Hennigs JK, Miyagawa K, Li CG, Nickel NP, Kaschwich M, Cao A, Wang L, Reddy S, Chen PI, Nakahira K, Alcazar MA, Hopper RK, Ji L, Feldman BJ, Rabinovitch M. BMPR2 preserves mitochondrial function and DNA during reoxygenation to promote endothelial cell survival and reverse pulmonary hypertension. Cell Metab. 2015;21(4):596-608. https://doi. org/10.1016/j.cmet.2015.03.010.

8. Sa S, Gu M, Chappell J, Shao NY, Ameen M, Elliott KA, Li D, Grubert F, Li CG, Taylor S, Cao A, Ma Y, Fong R, Nguyen L, Wu JC, Snyder MP, Rabinovitch M. Induced pluripotent stem cell model of pulmonary arterial hypertension reveals novel gene expression and patient specificity. Am J Respir Crit Care Med. 2017;195(7):930-41. https://doi.org/10.1164/ recm.201606-1200OC.

9. Ranchoux B, Antigny F, Rucker-Martin C, Hautefort A, Pechoux C, Bogaard HJ, Dorfmuller P, Remy S, Lecerf F, Plante S, Chat S, Fadel E, Houssaini A, Anegon I, Adnot S, Simonneau G, Humbert M, Cohen-Kaminsky S, Perros F. Endothelial-to-mesenchymal transition in pulmonary hypertension. Circulation. 2015;131(11):1006-18. https://doi.org/10.1161/ CIRCULATIONAHA.114.008750.

10. Masri FA, Xu W, Comhair SA, Asosingh K, Koo M, Vasanji A, Drazba J, Anand-Apte B, Erzurum SC. Hyperproliferative apoptosis-resistant endothelial cells in idiopathic pulmonary arterial hypertension. Am J Physiol Lung Cell Mol Physiol. 2007;293(3):L548-54. https://doi. org/10.1152/ajplung.00428.2006.

11. Tu L, De Man FS, Girerd B, Huertas A, Chaumais MC, Lecerf F, Francois C, Perros F, Dorfmuller P, Fadel E, Montani D, Eddahibi S, Humbert M, Guignabert C. A critical role for p130Cas in the progression of pulmonary hypertension in humans and rodents. Am J Respir Crit Care Med. 2012;186(7):666-76. https://doi.org/10.1164/rccm.201202-0309OC.

12. Tu L, Dewachter L, Gore B, Fadel E, Dartevelle P, Simonneau G, Humbert M, Eddahibi S, Guignabert C. Autocrine fibroblast growth factor-2 signaling contributes to altered endothelial phenotype in pulmonary hypertension. Am J Respir Cell Mol Biol. 2011;45(2):311-22. https:// doi.org/10.1165/rcmb.2010-0317OC.

13. Tu L, Guignabert C. Emerging molecular targets for anti-proliferative strategies in pulmonary arterial hypertension. Handb Exp Pharmacol. 2013;218:409-36. https://doi. org/10.1007/978-3-642-38664-0_17.

14. Ma L, Roman-Campos D, Austin ED, Eyries M, Sampson KS, Soubrier F, Germain M, Tregouet DA, Borczuk A, Rosenzweig EB, Girerd B, Montani D, Humbert M, Loyd JE, Kass RS, Chung WK. A novel channelopathy in pulmonary arterial hypertension. N Engl J Med. 2013;369(4):351-61. https://doi.org/10.1056/NEJMoa1211097.

15. Guignabert C, Bailly S, Humbert M. Restoring BMPRII functions in pulmonary arterial hypertension: opportunities, challenges and limitations. Expert Opin Ther Targets. 2017;21(2):18190. https://doi.org/10.1080/14728222.2017.1275567.

16. Morrell NW, Bloch DB, ten Dijke P, Goumans MJ, Hata A, Smith J, Yu PB, Bloch KD. Targeting BMP signalling in cardiovascular disease and anaemia. Nat Rev Cardiol. 2016;13(2):106-20. https://doi.org/10.1038/nrcardio.2015.156.

17. Spiekerkoetter E, Sung YK, Sudheendra D, Scott V, Del Rosario P, Bill M, Haddad F, LongBoyle J, Hedlin H, Zamanian RT. Randomised placebo-controlled safety and tolerability trial of FK506 (tacrolimus) for pulmonary arterial hypertension. Eur Respir J. 2017;50(3). https:// doi.org/10.1183/13993003.02449-2016.

18. Drake KM, Dunmore BJ, McNelly LN, Morrell NW, Aldred MA. Correction of nonsense BMPR2 and SMAD9 mutations by ataluren in pulmonary arterial hypertension. Am J Respir Cell Mol Biol. 2013;49(3):403-9. https://doi.org/10.1165/rcmb.2013-0100OC.

19. Long L, Ormiston ML, Yang X, Southwood M, Graf S, Machado RD, Mueller M, Kinzel B, Yung LM, Wilkinson JM, Moore SD, Drake KM, Aldred MA, Yu PB, Upton PD, Morrell NW. Selective enhancement of endothelial BMPR-II with BMP9 reverses pulmonary arterial hypertension. Nat Med. 2015;21(7):777-85. https://doi.org/10.1038/nm.3877.

20. Tu L, Desroches-Castan A, Mallet C, Guyon L, Cumont A, Phan C, Robert F, Thuillet R, Bordenave J, Sekine A, Huertas A, Ritvos O, Savale L, Feige JJ, Humbert M, Bailly S, Guignabert C. Selective BMP-9 Inhibition Partially Protects Against Experimental Pulmonary Hypertension. Circ Res. 2019;124(6):846-55. https://doi.org/10.1161/CIRCRESAHA.118.313356. 
21. Ricard N, Tu L, Le Hiress M, Huertas A, Phan C, Thuillet R, Sattler C, Fadel E, Seferian A, Montani D, Dorfmuller P, Humbert M, Guignabert C. Increased pericyte coverage mediated by endothelial-derived fibroblast growth factor- 2 and interleukin- 6 is a source of smooth muscle-like cells in pulmonary hypertension. Circulation. 2014;129(15):1586-97. https://doi. org/10.1161/CIRCULATIONAHA.113.007469.

22. Michelakis ED, Gurtu V, Webster L, Barnes G, Watson G, Howard L, Cupitt J, Paterson I, Thompson RB, Chow K, O'Regan DP, Zhao L, Wharton J, Kiely DG, Kinnaird A, Boukouris AE, White C, Nagendran J, Freed DH, Wort SJ, Gibbs JSR, Wilkins MR. Inhibition of pyruvate dehydrogenase kinase improves pulmonary arterial hypertension in genetically susceptible patients. Sci Transl Med. 2017;9(413). https://doi.org/10.1126/scitranslmed.aao4583.

23. Huertas A, Perros F, Tu L, Cohen-Kaminsky S, Montani D, Dorfmuller P, Guignabert C, Humbert M. Immune dysregulation and endothelial dysfunction in pulmonary arterial hypertension: a complex interplay. Circulation. 2014;129(12):1332-40. https://doi.org/10.1161/ CIRCULATIONAHA.113.004555.

24. Huertas A, Tu L, Thuillet R, Le Hiress M, Phan C, Ricard N, Nadaud S, Fadel E, Humbert M, Guignabert C. Leptin signalling system as a target for pulmonary arterial hypertension therapy. Eur Respir J. 2015;45(4):1066-80. https://doi.org/10.1183/09031936.00193014.

25. Huertas A, Tu L, Gambaryan N, Girerd B, Perros F, Montani D, Fabre D, Fadel E, Eddahibi S, Cohen-Kaminsky S, Guignabert C, Humbert M. Leptin and regulatory T-lymphocytes in idiopathic pulmonary arterial hypertension. Eur Respir J. 2012;40(4):895-904. https://doi. org/10.1183/09031936.00159911.

26. Tamura Y, Phan C, Tu L, Le Hiress M, Thuillet R, Jutant EM, Fadel E, Savale L, Huertas A, Humbert M, Guignabert C (2018) Ectopic upregulation of membrane-bound IL6R drives vascular remodeling in pulmonary arterial hypertension. J Clin Invest 128 (5):1956-1970. https:// doi.org/10.1172/JCI96462.

Open Access This chapter is licensed under the terms of the Creative Commons Attribution 4.0 International License (http://creativecommons.org/licenses/by/4.0/), which permits use, sharing, adaptation, distribution and reproduction in any medium or format, as long as you give appropriate credit to the original author(s) and the source, provide a link to the Creative Commons license and indicate if changes were made.

The images or other third party material in this chapter are included in the chapter's Creative Commons license, unless indicated otherwise in a credit line to the material. If material is not included in the chapter's Creative Commons license and your intended use is not permitted by statutory regulation or exceeds the permitted use, you will need to obtain permission directly from the copyright holder.

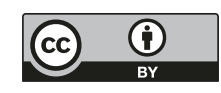

